Check for updates

Cite this: RSC Adv., 2019, 9, 28199

Received 13th August 2019

Accepted 26th August 2019

DOI: $10.1039 / c 9 r a 06285 h$

rsc.li/rsc-advances

\section{Pd nanoparticles on green support as dip-catalyst: a facile transfer hydrogenation of olefins and $\mathrm{N}$ - heteroarenes in water $\uparrow$}

\author{
M. Nasiruzzaman Shaikh iD
}

Chemo- and regioselective hydrogenation methods using highly green sources, particularly from metal nanoparticles on plant stem as support and water, is an intensive research area, which is highly relevant to the development of green chemistry and technology in the $21^{\text {st }}$ century. Here, the synthesis and activity of a heterogeneous catalytic system (called "dip-catalyst") for the transfer hydrogenation of a series of styrenyl, unfunctionalized olefins, quinoline and other $N$-heteroarenes, are presented. It consists of Pd nanoparticles (15-20 nm) anchored on bio-processed jute plant (Corchorus genus) stem as the support. Pd nanoparticles were decorated on the green support (GS) jute stem by the in situ reduction of $\mathrm{K}_{2} \mathrm{PdCl}_{4}$ in aqueous medium at $70{ }^{\circ} \mathrm{C}$, using formic acid as the reductant. The Pd@GS was characterized using SEM, EDS, XRD, FTIR, XPS and TEM. Elemental mapping revealed uniform distribution of $\mathrm{Pd}$ on the cellulose matrix of the jute stem. The catalyst was successfully applied to the chemoselective transfer hydrogenation of numerous styrenyl, unfunctionalized olefins. Its high functional group tolerance was investigated during the olefins hydrogenation in water. Furthermore, PdaGS was capable of quantitative hydrogenation to selectively produce 1,2,3,4-tetrahydroquinoline (THQ) in water using stoichiometric amounts of tetrahydroxydiboron (THDB) at $60{ }^{\circ} \mathrm{C}$ with turn over frequency (TOF) $4938 \mathrm{~h}^{-1}$. This system is stable in water and displays excellent recyclability; it could be used for 32 consecutive cycles, without losing its original crystallinity or requiring replenishment.

\section{Introduction}

Transition metal-mediated hydrogenation ${ }^{\mathbf{1}, 2}$ using $\mathrm{Fe},{ }^{3} \mathrm{Co},{ }^{4} \mathrm{Ni},{ }^{5}$ $\mathrm{Pd},{ }^{6,7} \mathrm{Pt},{ }^{8} \mathrm{Rh},{ }^{9,10} \mathrm{Ir}^{11}{ }^{12} \mathrm{Ru}^{12}$ or $\mathrm{Mo}^{13}$ is a well-established procedure in research and industrial applications. However, this approach usually requires high hydrogen pressure, resulting in over reduction or functional group intolerance of the substrate. To overcome this shortcoming, transfer hydrogenation ${ }^{\mathbf{1 4 , 1 5}}$ is an alternative. In this context, considerable efforts have been devoted to design Pd-supported transfer hydrogenation homogeneous catalysts. ${ }^{16-18}$ Since these catalysts are well dispersed at the molecular level, every individual catalytic entity can act as an active and accessible mechanistic site to which the reactant is exposed. This leads to an overall high catalytic efficiency. Unfortunately, they suffer from some serious drawbacks such as cumbersome separation, usage of high volume of toxic solvents and continuous loss of precious metal. ${ }^{19}$ Furthermore, their restricted reusability impede them from wide commercialization. Nevertheless, the current environmental related

Center of Research Excellence in Nanotechnology (CENT), King Fahd University of Petroleum and Minerals (KFUPM), Dhahran, Saudi Arabia. E-mail: mnshaikh@ kfupm.edu.sa

$\dagger$ Electronic supplementary information (ESI) available. See DOI: 10.1039/c9ra06285h governmental legislation imposes severe restriction to eliminate toxic chemical processing and move towards the safer and greener technological endeavours.

To overcome these challenges, immobilization of the catalyst is one of the obvious choices. It is well known that the activity of heterogeneous catalytic systems rely heavily on the nature of support. ${ }^{20}$ Hence, a prudent choice of support is vital. Silica, ${ }^{21,22}$ polymers, ${ }^{23,24}$ zeolites, ${ }^{25,26}$ alumina, ${ }^{27,28}$ magnetic nanoparticles, ${ }^{29,30}$ and carbon $^{31}$ have emerged as excellent support systems. They reportedly achieve almost quantitative conversion and selectivity. Furthermore, their selection is an artifact of particle size, shape and porosity. ${ }^{32}$ However, due to small size and high surface energy, the active metal nanoparticles tend to aggregate on the support, thereby lowering the number of accessible sites for the reactant; this results in low catalytic activity and selectivity. Moreover, facile leaching of nanoparticles from the surface of the support, due to weak binding, restricts the prospect of reusability. ${ }^{33}$

Recently, a great deal of attention has been devoted to develop solid supports from biomass, such as activated carbon, carbon nanofibers and hydrophilic carbons, as they are treated as sustainable support materials in heterogeneous catalysis. ${ }^{34}$ Among these, activated carbon supports were widely used as metal nanoparticle anchors for catalytic applications; however, they yielded relatively discouraging performance. ${ }^{35}$ The major 
reasons of failure could be (1) poor pore connectivity, resulting in limited mass-transfer and longer diffusion times, (2) lack of proper functional group on the support, hence hindering the metal nanoparticles' interaction and, (3) hydrophobic surface.

In this context, a new concept of "dip-catalyst" has emerged in order to achieve better efficiency, high turnover frequency (TOF), ease of fabrication, higher reusability and greener synthesis routes. ${ }^{36,37}$ The term "dip-catalyst" refers to the convenient deployment, high recyclability, easy insertion/ removal which can turn the reaction on/off almost instantaneously. The area of "dip-catalysis" has remained largely unexplored. Recently, a few reports related to the fabrication of "dip-catalyst" have appeared in the literature. ${ }^{38}$ Hariprasad et al. reported Pd-PVA based thin films with high catalytic activity and reusability. ${ }^{39}$ Zheng et al. reported a paper-based composite material consisting of metal ions on filter paper, and achieved excellent activity and recyclability. ${ }^{40}$ Rosa et al. dispersed Pd nanoparticles on cellulose acetate and successfully applied it for the $\mathrm{C}-\mathrm{C}$ bond formation reaction. ${ }^{41}$

Encouraged by such distinctive features of having highly improved activity and excellent recyclability over the conventional support-based catalysts, we continue to contribute to the development of highly recyclable heterogeneous catalysts. ${ }^{29,42-44}$ Here, we report a "dip-catalyst" comprising Pd nanoparticles supported on bio-processed jute stem (Pd@GS), for the transfer hydrogenation of olefins, quinoline and other $N$-heteroarenes in water (Fig. 1). The excellent conversion and selectivity over sensitive organic functional group and its reusability for tens of consecutive cycles was studied systematically.

\section{Experimental}

\section{Materials and methods}

All chemicals were purchased from Sigma-Aldrich and were used as-received unless otherwise stated. Standard procedures were followed for the dry and deoxygenated solvents. Schlenk line techniques were used to carry out reactions under inert

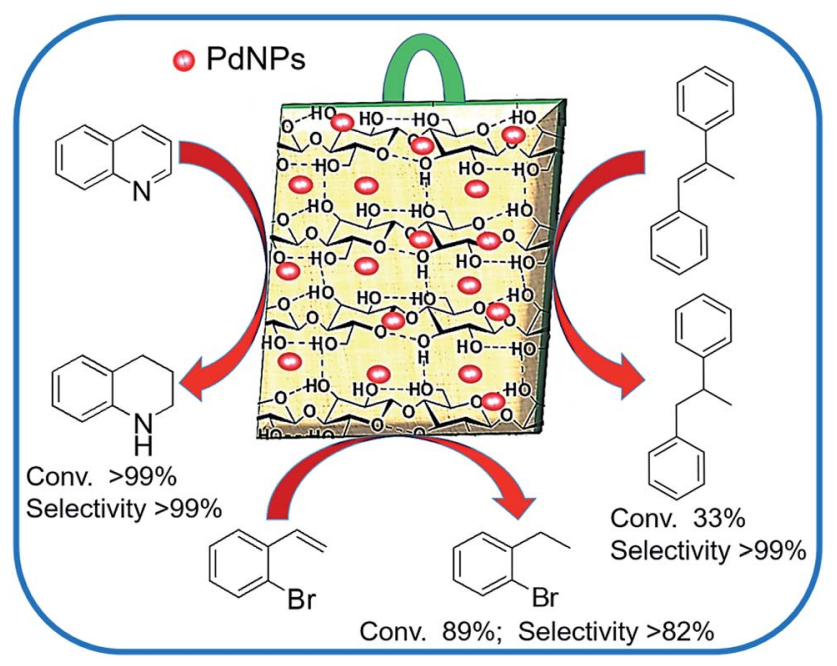

Fig. 1 Graphical presentation of versatility of Pd (aGS as "dip catalyst". atmosphere wherever needed. Deionized (DI) water (specific conductivity: $18.2 \mathrm{M} \Omega$ ) was used in all the experiments. FourierTransform Infrared (FTIR) spectroscopic data were recorded on a Nicolet 720 in the wave number range of 500 to $4000 \mathrm{~cm}^{-1}$, using ATR. The TEM samples were prepared by dropping an ethanolic suspension on a copper grid and drying at room temperature. The amount of Pd in the catalyst was determined by inductively coupled plasma optical emission spectrometry (ICP-OES; PlasmaQuant PO 9000 - Analytik Jena). The samples were first digested in a dilute mixture of $\mathrm{HNO}_{3}$ and $\mathrm{HCl}$. Calibration curves were prepared for Pd using standard solutions (ICP Element Standard Solutions, Merck). Samples for SEM (Lyra 3, Tescan) imaging were prepared from a chunk of decorated jute stem on alumina stubs and coated with gold in an automatic gold coater (Quorum, Q150T E). For elemental analysis and mapping, the energy dispersive X-ray spectra (EDS) were collected on a Lyra 3 attachment, using an Oxford Instruments. Catalytic products were identified by a Shimadzu 2010 Plus gas chromatograph attached with a mass spectrometer (GC-MS, Japan). The disappearance of the reactant and sequential appearance of the product was recorded in real time, identifying the species in terms of their molecular ion $\left(\mathrm{M}^{+}\right)$by comparing and matching them with the available Wiley library of the mass spectrum database, in addition to the identification of mass fragments.

\section{Synthesis of Pd@GS}

Usually, the jute plants are harvested after they bloom. The fully grown stalks are cut, trimmed, bundled and kept immersed in ponds or stream to rot (known as retting). This frees the fibers from the stem, which are removed manually. The stems are dried for 7 days in sunlight, cut into thin slices $(\sim 2 \mathrm{~cm} \times 0.5 \mathrm{~cm}$ $\times 0.1 \mathrm{~cm})$ and dried in an oven at $100{ }^{\circ} \mathrm{C}$ for $5 \mathrm{~h}$. Pd nanoparticles in the range of 15-20 nm on jute stem were prepared as described below and its schematic illustration is shown in Fig. 2. The oven dried jute-stem thin slices of the above dimensions were immersed in $\mathrm{K}_{2} \mathrm{PdCl}_{4}$ solution in DI water (32 $\mathrm{mg} / 20 \mathrm{~mL}$ ) and soaked for $2 \mathrm{~h}$. The vial containing the suspension was heated at $70{ }^{\circ} \mathrm{C}$ for $1 \mathrm{~h}$. $100 \mu \mathrm{L}$ formic acid was added to reduce the Pd precursors while the mixture was stirred for $15 \mathrm{~min}$ and the color changed to black. Heating was continued for another hour to complete the reduction and lodging of the nanoparticles onto the substrate. The contents were allowed to settle down. The thin jute slices completely
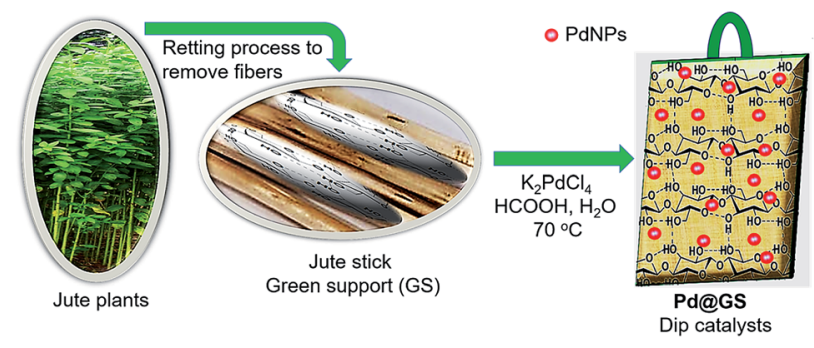

Fig. 2 Schematic illustration for the synthesis of PdaGS 
covered with black Pd nanoparticles were pulled out (Fig. 3a). They were dried in open air for $24 \mathrm{~h}$ and samples were prepared for characterization.

\section{Olefin hydrogenation}

The catalytic hydrogenation was performed in a $20 \mathrm{~mL}$ screwcapped vial under the conditions stated below. To the suspension of tetrahydroxydiboron (THDB, $3.1 \mathrm{mmol}$ ) in $5 \mathrm{~mL}$ water, the substrate $(1 \mathrm{mmol})$ was added and stirred. The vial was capped and introduced into a pre-heated $\left(70^{\circ} \mathrm{C}\right)$ oil bath. After thermal equilibrium, the Pd@GS strip was added to the vial, which was stirred magnetically. The progress of the reaction was monitored by TLC (thin layer chromatography) and GC by periodically withdrawing small aliquots from the vial. The product was extracted in dichloromethane (DCM), dried with magnesium sulfate and passed through short silica gel column. The conversion and selectivity were determined by GC and identified and quantified by GC-MS.

\section{Quinoline hydrogenations}

The transfer hydrogenation of quinoline was conducted in a $10 \mathrm{~mL}$ glass reaction tube reactor fitted with magnetic stirrer and a Teflon screw cap. THDB $(4.1 \mathrm{mmol})$, quinoline $(1 \mathrm{mmol})$ and DI water $(5 \mathrm{~mL})$ were added and stirred @ $550 \mathrm{rpm}$. A strip of Pd@GS catalyst was inserted and stirred at $60{ }^{\circ} \mathrm{C}$ at the same speed. The progress of the reaction was monitored by TLC and GC as in olefin hydrogenation. After cooling, the reaction mixture was extracted with DCM, dried with magnesium sulfate and the solvent removed under vacuum. The residue was redissolved in minimum amount of DCM and passed through a short silica gel column using a mixture of hexane and ethyl acetate $(8: 2)$ as the eluent. GC was used to measure the conversion and selectivity and the products were identified by GC-MS.

\section{Results and discussion}

\section{Synthesis and characterization}

The fully grown jute was cut, trimmed. Stems were extracted through the retting process. Since, the jute stick is endowed with highly fibrous, porous, lightweight, voluminous and woody structure, it has extremely high surface area. About 3 million tons of jute sticks are produced annually in India; it is economically cheap, affordable and a biodegradable green

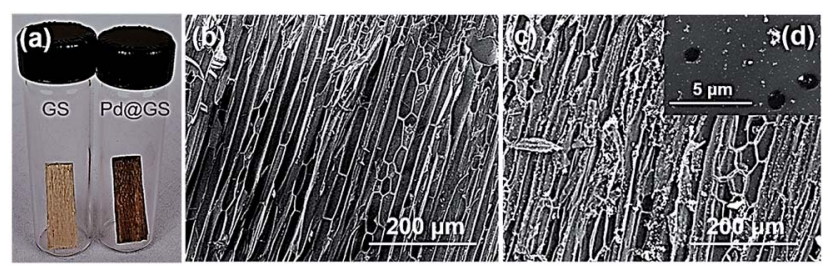

Fig. 3 Images of (a) the pure GS and prepared PdaGS catalyst and SEM images of (b) pure green support (c) PdaGS; inset (d) is a magnified view showing uniform distribution of Pd nanoparticles. precursor. The jute stem is a lignocellulosic raw material, composed of $\alpha$-cellulose $(40.8 \%)$, hemicellullose $(22.10 \%)$, lignin (23.5\%), and others. ${ }^{45}$ Anchoring of Pd nanoparticles was achieved by immersing the jute strips, which contain large number of primary and secondary hydroxyl groups, into potassium tetrachloropalladate $\left(\mathrm{K}_{2} \mathrm{PdCl}_{4}\right)$ solution in DI water and allowing it to be sufficiently soaked in the metal precursor solution for $2 \mathrm{~h}$. The yellow palladium precursor was reduced in situ to black Pd nanoparticles by using formic acid as reductant at $70{ }^{\circ} \mathrm{C}$.

The morphological and nanostructural features of pure jute stem and Pd@GS are revealed in SEM images shown in Fig. 3. Pure jute strips are highly fibrous - a characteristic that remained intact after the decoration with Pd nanoparticles via in situ reduction in the acidic media. Formation of spherical Pd nanoparticles with uniform distribution all over the strips is also evident. This was further corroborated by confined area elemental mapping and EDS quantification, as shown in Fig. 4.

TEM images show uniform distribution of Pd with average diameter of 15-20 nm (Fig. 5a and b), which was further confirmed by measuring the particles size using dynamic light scattering technique. The selected area electron diffraction (SAED) image is shown in Fig. 5c. The calculated $d$-value could be assigned to the $\langle 111\rangle$ reflection of the $f c c$ structured Pd nanoparticles. ${ }^{46}$

FTIR spectroscopic technique was used to evaluate the decoration. The abundant number of hydroxyl group on the pure jute stem were detected by a characteristic peak at around $3345 \mathrm{~cm}^{-1}$; there was no significant change in the signature after Pd decoration (see ESI $\dagger$ ).

The XPS analysis was performed on the as-synthesized Pd@GS to identify the electronic states of Pd. The overall composition is depicted in the global XPS survey (Fig. 6a) which confirmed the presence of $\mathrm{C}, \mathrm{O}$ and $\mathrm{Pd}$. Formation of $\mathrm{Pd}^{0}$ on the surface of jute stem is confirmed by the deconvolution in the Pd doublet peaks (Fig. 6b) with binding energies of $335.3 \mathrm{eV}\left(3 \mathrm{~d}_{5 / 2}\right)$
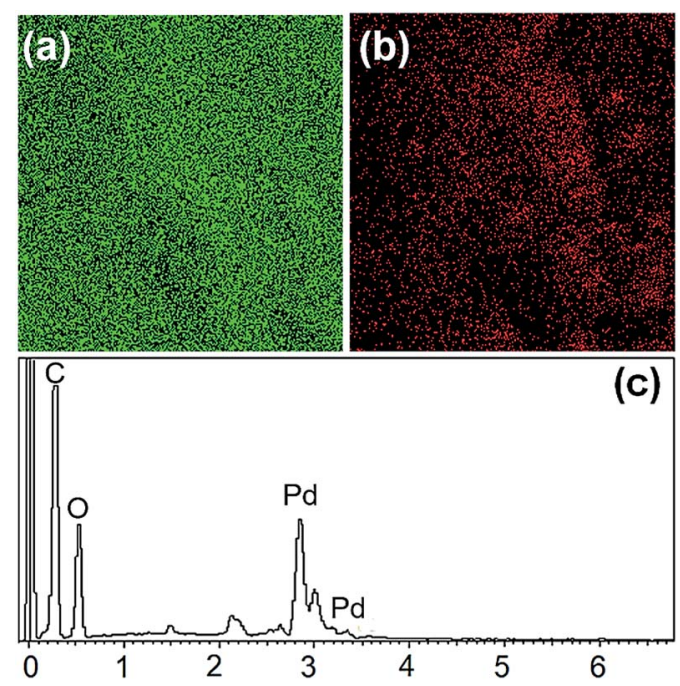

Fig. 4 Elemental mapping of (a) carbon and (b) Pd. The EDS signature of the PdaGS catalyst is shown in (c). 

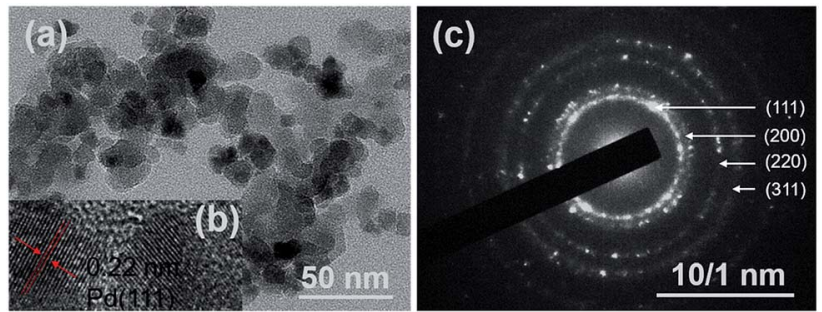

Fig. 5 (a) TEM image of Pd nanoparticles anchored on the surface (b) HRTEM image and (c) SAED of PdaGS.
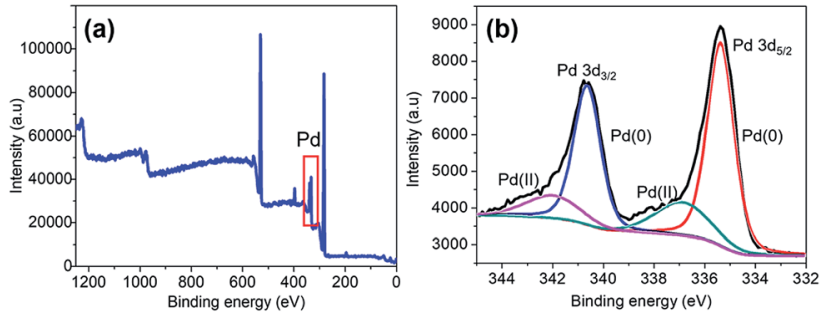

Fig. 6 XPS signatures of PdaGS: (a) the survey data and (b) oxidation states of Pd decorating the support surface.

and $340.5 \mathrm{eV}\left(3 \mathrm{~d}_{3 / 2}\right)$ associated with $\mathrm{Pd}^{0}{ }^{0}{ }^{47}$ peaks at $337.2 \mathrm{eV}$ and $342.4 \mathrm{eV}$ are associated with $\mathrm{Pd}^{2+}$ due to the formation of PdO, probably resulting from partial oxidation of the Pd nanoparticles at the ambient. ${ }^{48}$

The Pd content of each strip was quantified by the ICP-OES technique. The optimum amount of palladium nanoparticles was measured by varying the concentration of the $\mathrm{K}_{2} \mathrm{PdCl}_{4}$ solutions. The Pd content was found to increase with higher concentration of pre-reduced Pd and then remained constant with an average Pd content determined to be $0.0288 \pm 0.008 \mathrm{mg}$ per strip. Hence, the reproducibility of the Pd nanoparticles loading imply the natural limit to its highly hydroxylated surface. This higher loading could be attributed to the combination of strong van der Waals forces and hydrophobic interaction between metal nanoparticles and the jute-stick surface, leading to the nanoparticles immobilization. ${ }^{49,50}$

\section{Catalysis}

Styrenyl alkenes hydrogenation. Results on the hydrogenation of a number of differently substituted olefins are summarized in Table 1. The optimization reactions were performed with styrene as the benchmark substrate, by varying the temperature, solvents, stoichiometry of reductant, and the duration. Styrene was $77 \%$ converted into its corresponding hydrogenated product in $1 \mathrm{~h}$ at $70{ }^{\circ} \mathrm{C}$ in water (as solvent) with the 3.1 equivalent of THDB (entry\# 1 ) with maximum selectivity. Lowering the temperature to $50{ }^{\circ} \mathrm{C}$ decreased the conversion to $48 \%$ (entry\# 2) with lower TOF $889 \mathrm{~h}^{-1}$ compared to $2851 \mathrm{~h}^{-1}$ at $70{ }^{\circ} \mathrm{C}$. Changing the solvent to methanol promoted the same reaction with limited conversion (33\%). In the case of a blank reaction in presence of Pd@GS in water but without THDB, no olefin hydrogenation was detected; in the presence of THDB (3.1 equiv.) without catalyst, about $<8 \%$ of styrene was converted to ethylbenzene (entry\# 4). The role of support in the hydrogenation process was investigated. In absence of Pd and THDB, no conversion was noted (entry\# 5).

Wide applicability of the catalyst was examined by employing styrene with various functional groups - both electron donating and electron withdrawing groups. No significant effect of the electron donating or electron withdrawing groups was seen in the case of styrenyl olefins. For example, $p$-methyl styrene and $p$-vinyl anisole were quantitatively hydrogenated with maximum selectivity (entries\# 6 and 7). A similar trend was observed with choloro-substituted styrene (entry\# 8); no dehalogenation was observed. The reactivity of Pd@GS towards the reduction of $m$-nitrostyrene (where under similar reaction conditions, both olefin and nitro functional groups are prone to simultaneous reduction) was also evaluated. At $45{ }^{\circ} \mathrm{C}$, the conversion was complete but non-selective, where both functional groups were completely and simultaneously reduced in $20 \mathrm{~h}$, in water.

Selectivity against two or more competitive functional groups toward hydrogenation process is highly challenging. Reaction conditions, including choice of solvent, need critical optimization. In DCM, $82 \%$ of the $m$-nitrostyrene was converted and the selectivity towards $m$-nitroethylbenzene reached up to $60 \%$ while for the remaining $40 \%$ both nitro and olefin functional groups were completely reduced to form $\mathrm{m}$-aminoethylbenzene, at $45^{\circ} \mathrm{C}$ with the use of 2.1 equivalent of THDB as reductant.

Under similar reaction conditions with THF the overall conversion was $88 \%$ with significant enhancement in selectivity (72\%) towards $m$-nitroethylbenzene (entry\# 11). The effect of temperature and variation of mole equivalent of THDB in selectivity were also evaluated in THF. The results are shown in Fig. 7a. On increasing the mole equivalent of THDB from 1.1 to 4.1 , the conversion increased from $48 \%$ to $>99 \%$. However, the selectivity drastically reduced from $\sim 81 \%$ to $\sim 47 \%$ for the 4.1 mole equivalent of THDB. These results are instructive in that reaction parameters can be subtly tweaked to reduce the alkenyl group in styrene in the presence of an easily reducible nitro functional group with very high selectivity.

Another interesting example is the reduction of $o$-bromostyrene to form the corresponding olefin hydrogenated product (cf. Fig. 7b), $o$-bormoethylbenzene at $40{ }^{\circ} \mathrm{C}$. In most cases, hydrogenation of bromostyrene occurs via either hydrogenolysis, where dehalogenation is obviated, or through polymerization, or both take place simultaneously. Yet, it is to our delight that our Pd@GS catalyst demonstrated excellent reactivity and selectivity without hydrogenolysis nor polymerization. In water as solvent, at $40{ }^{\circ} \mathrm{C}, o$-bromostyrene yielded only ethylbenzene with complete debromination in $24 \mathrm{~h}$ (entry\# 13). But when the reaction was performed at $40{ }^{\circ} \mathrm{C}$ in DCM with 1.1 equivalent of THDB the conversion achieved was about $40 \%$ in $48 \mathrm{~h}$ with very high (75\%) selectivity towards the formation of $o$ bromoethylbenzene and significant amount of $(22 \%)$ ethylbenzene with $3 \%$ of styrene was also formed (entry\# 14) as side product. By changing the solvent to THF, the conversion was significantly improved to $89 \%$ and the highest selectivity (82\%) 
Table 1 Hydrogenation of PdaGS catalyzed styrene and its derivatives with THDB in different solvents

\begin{tabular}{|c|c|c|c|c|c|c|c|c|}
\hline Entry & Subst. $^{a}(\mathrm{R})$ & Solv. & THDB (equiv.) & Temp. $\left({ }^{\circ} \mathrm{C}\right)$ & Time (h) & Conv. $^{b}(\%)$ & $\operatorname{Sel}^{c}(\%)$ & TOF $\left(\mathrm{h}^{-1}\right)$ \\
\hline 1 & \multirow[t]{5}{*}{$\mathrm{H}$} & $\mathrm{H}_{2} \mathrm{O}$ & 3.1 & 70 & 1 & 77 & $>99$ & 2851 \\
\hline 2 & & $\mathrm{H}_{2} \mathrm{O}$ & 3.1 & 50 & 2 & 48 & 99 & 889 \\
\hline 3 & & $\mathrm{MeOH}$ & 3.1 & 70 & 1 & 30 & $>99$ & 1111 \\
\hline $4^{d}$ & & $\mathrm{H}_{2} \mathrm{O}$ & 3.1 & 70 & 1 & $<8$ & nd & nd \\
\hline $5^{e}$ & & $\mathrm{H}_{2} \mathrm{O}$ & 3.1 & 70 & 10 & nd & nd & nd \\
\hline 6 & $p-\mathrm{CH}_{3}$ & $\mathrm{H}_{2} \mathrm{O}$ & 3.1 & 70 & 1 & $>99$ & $>99$ & 3704 \\
\hline 7 & $p-\mathrm{OCH}_{3}$ & $\mathrm{H}_{2} \mathrm{O}$ & 3.1 & 70 & 1 & 98 & $>99$ & 3630 \\
\hline 8 & $p$-Cl & $\mathrm{H}_{2} \mathrm{O}$ & 3.1 & 70 & 1 & $>99$ & $>99$ & 3704 \\
\hline 9 & \multirow[t]{4}{*}{$m-\mathrm{NO}_{2}$} & $\mathrm{H}_{2} \mathrm{O}$ & 3.1 & 45 & 20 & $>99$ & $<2$ & 185 \\
\hline 10 & & THF & 1.1 & 45 & 24 & 48 & 81 & 74 \\
\hline 11 & & THF & 2.1 & 45 & 24 & 88 & 72 & 136 \\
\hline 12 & & DCM & 2.1 & 45 & 24 & 82 & 60 & 126 \\
\hline 13 & \multirow[t]{4}{*}{$o-\mathrm{Br}$} & $\mathrm{H}_{2} \mathrm{O}$ & 2.1 & 40 & 24 & $>99$ & $<5$ & 154 \\
\hline 14 & & DCM & 1.1 & 40 & 48 & 40 & 75 & 31 \\
\hline 15 & & THF & 1.1 & 40 & 48 & 89 & 82 & 69 \\
\hline 16 & & THF & 1.1 & 50 & 48 & 93 & 67 & 77 \\
\hline
\end{tabular}

${ }^{a} 1 \mathrm{mmol}$ of substrate with $3.1 \mathrm{mmol}$ THDB with Pd@GS as catalyst. ${ }^{b}$ Measured by GC. ${ }^{c}$ Identified by GC-MS. ${ }^{d}$ Without Pd@GS but with 3 equivalent of THDB; nd-not determined. ${ }^{e}$ Conducted reaction using only GS.

towards $o$-bromoethylbenzene was achieved (entry\# 15). To the best of our understanding, it is highest selectivity available in literature under such very mild reaction conditions. On increasing the temperature at $50{ }^{\circ} \mathrm{C}$, the conversion was slightly improved to $93 \%$ with the sacrifice of selectivity, which reached up to $67 \%$-bromoethylbenzene and $33 \%$ de-brominated product (entry\# 16).

Nonetheless, this reaction could be tuned to synthesize bromoalkyl benzene from bromostyrene and at the same time it is also worthy to mention that debrominated alkylbenzene, which bears significant value in literature, ${ }^{51}$ can also be prepared from the bromostyrene by simply changing the reaction condition using this catalytic system. This dual nature of the catalyst may mark it as a practical catalyst.

Cyclic and acylic polysubstituted alkenes hydrogenation. There are large number of olefin substrates that resist hydrogenation by conventional catalysts; ${ }^{52}$ unfunctionalized olefins fall within this class. However, breakthrough has been made by Stokes and co-workers who demonstrated excellent reactivity of Pd-based catalyst which could catalyze a variety of unfunctionalized olefins. ${ }^{53}$

Following that report, we employed various olefins to test the wide applicability of Pd@GS. The results are shown in Table 2 which summarizes the comparison of reactivity of Pd@GS towards differently substituted linear and cyclic alkenes. For instance, cyclic alkene (entry\# 1) was hydrogenated in THF at $60{ }^{\circ} \mathrm{C}$ in $20 \mathrm{~h}$ with $49 \%$ conversion. Hydrogenation was also conducted with the tri-substituted alkenes such as trans- $\alpha$ methylstilbene (entry\# 2) and ethyl trans- $\beta$-methylcinnamate (entry\# 3) in THF at $70{ }^{\circ} \mathrm{C} .33 \%$ conversion was achieved with trans- $\alpha$-methylstilbene with maximum selectivity. Clear contrast was observed when the functionalized olefins, for instance methyl-2-acetamido propionate (entry\# 4), were employed for hydrogenation.

Quinoline hydrogenation. To assist the optimization process, quinoline was employed a model benchmark substrate for the catalytic hydrogen transfer reaction using Pd@GS as a catalyst and the progress of the reaction was monitored by TLC and GC, and the products were identified by GC-MS. First, blank reaction was carried out in water inserting the Pd@GS catalyst in the absence of THDB; reaction did not yield any hydrogenated product. When THDB was used without catalyst under identical reaction conditions, 25\% conversion was achieved. When both catalyst (Pd@GS) and THDB were used, the yield of 1,2,3,4-tetrahydroquinoline (py-THQ) reached $>99 \%$ with exclusive selectivity. Hence, a combination of catalyst and diboron is required to facilitate the transfer hydrogenation process. To explore the catalytic activity, the degree of sensitivity of the catalyst towards various parameter, such as solvent, temperature, amount of THDB and catalyst, was thoroughly evaluated and the results are shown in Table 3.
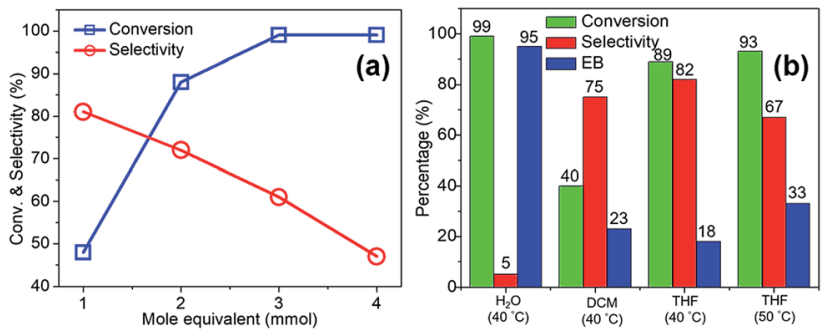

Fig. 7 (a) Variation in the conversion and selectivity of $m$-nitrostyrene at $45{ }^{\circ} \mathrm{C}$ with THDB mole equivalent in THF (b) conversion and selectivity of 0 -bromostyrene with 1.1 equivalent of THDB in different solvents. EB: ethylbenzene. 
Table 2 PdaGS catalyzed cyclic and acylic polysubstituted alkene hydrogenation in THF

\begin{tabular}{|c|c|c|c|c|c|c|c|}
\hline Entry & Substrate $^{a}$ & Product & Temp $\left({ }^{\circ} \mathrm{C}\right)$ & Time (h) & Conv. $^{b}(\%)$ & Sel. $^{c}(\%)$ & TOF $\left(h^{-1}\right)$ \\
\hline 1 & & & 60 & 20 & $>49$ & $>99$ & 91 \\
\hline 2 & & & 70 & 24 & 33 & $>99$ & 51 \\
\hline 3 & & & 70 & 24 & 7 & $>99$ & 11 \\
\hline 4 & & & 60 & 20 & $>99$ & $>99$ & 185 \\
\hline
\end{tabular}

${ }^{a} 1 \mathrm{mmol}$ with $4 \mathrm{mmol}$ THDB in THF as solvent and $2 \mathrm{~cm}$ long strip of catalyst, Pd@GS. ${ }^{b}$ Measured by GC. ${ }^{c}$ Identified by GC-MS.

When temperature was increased to $60{ }^{\circ} \mathrm{C}$, the reaction proceeded smoothly with $>99 \%$ conversion and $>99 \%$ selectivity in $40 \mathrm{~min}$. It can be observed that turn over frequency (TOF) was significantly higher (TOF $4938 \mathrm{~h}^{-1}$ ) compared to the TOF (1574 $\mathrm{h}^{-1}$ ) at lower temperature (entries\# 1 and 2). Changing solvent to DCM and THF affected the yield adversely; no hydrogenation occurred (entries\# 3 and 4). With methanol, 89\% conversion was achieved (entry\# 5). This could be ascribed to the participation of lone e-pair of the oxygen atom in $\mathrm{MeOH}$ solvent in the formation of boron adduct in the transition state. Conversion was improved with increasing amount of THDB and best results were obtained with 4.1 equivalent of THDB. It would seem that large amount of gaseous hydrogen escapes reaction medium without interacting with the metal to form metal-hydride intermediate, which is responsible for the hydrogenation of heterocyclic compounds. Under the above optimized reaction conditions, other $\mathrm{N}$-heterocycles were subjected to transfer hydrogenation reaction in water and smoothly converted to hydrogenated product contain nitrogen ring with maximum selectivity. But surprisingly, though isoquinoline was quantitatively hydrogenated with $>99 \%$ selectivity (entry\# 6), it took much longer time $(5 \mathrm{~h})$ than the quinoline. In case of polycyclic aromatic heterocycles, such as acridine, the reactions proceeded smoothly with excellent yield and selectivity (entry\# 7). Five and six membered ring compounds, pyrrole and pyrazine, were also employed as substrate and the catalyst Pd@GS, was found be highly reactive to convert the substrate into the hydrogenated product in water with TOF $1652 \mathrm{~h}^{-1}$ and $1815 \mathrm{~h}^{-1}$ respectively (entries\# 8 and 9). There was no conversion was observed only in presence of GS but in absence of PdNPs and THDB (entry\# 10). To compare the catalytic activity of Pd@GS with standard catalyst (entries\# 11-13), it was observed that our "dip-catalyst", Pd@GS, performance was having an edge in terms of reactivity and gain superiority in terms of re-usability over the standard catalyst used.

Table 3 Pd@GS catalyzed quinoline hydrogenation in water using THDB as reductant

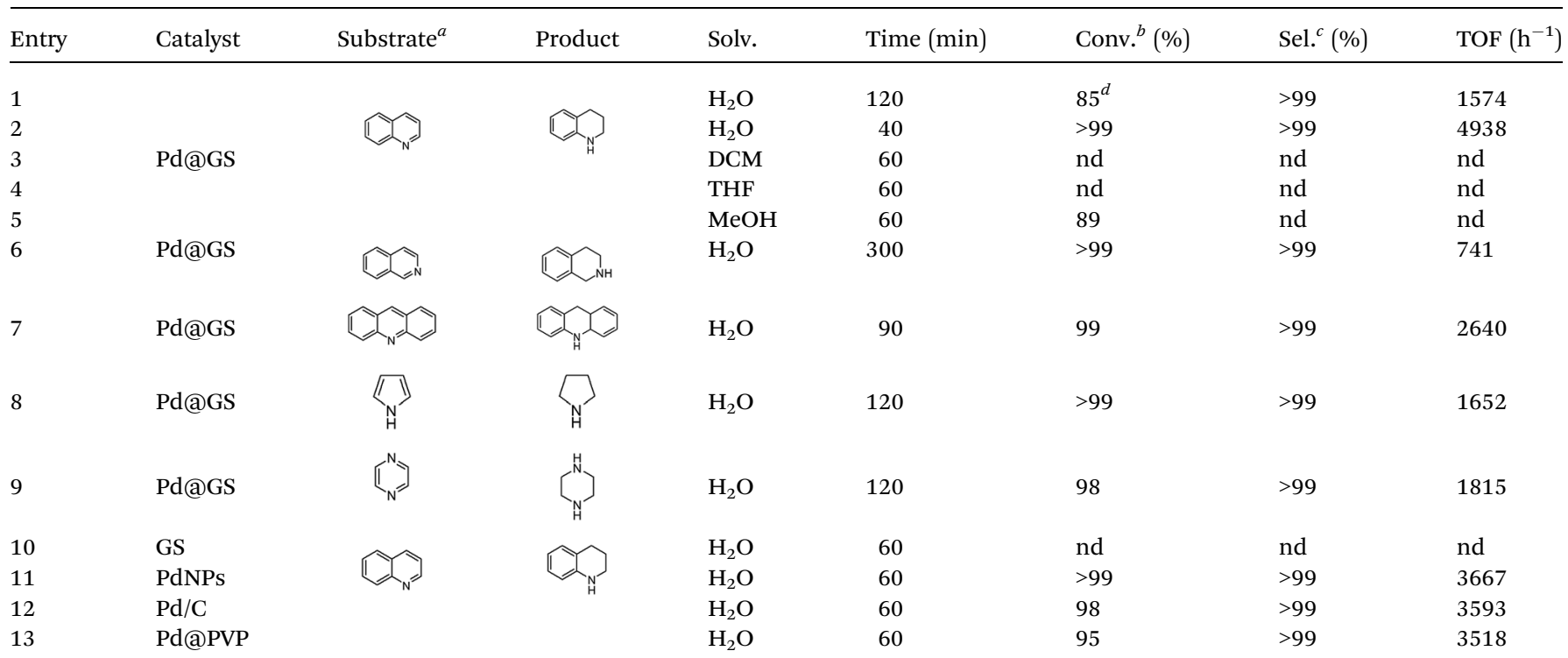

${ }^{a}$ Substrate used $1 \mathrm{mmol}$ and THDB $3.1 \mathrm{mmol}$ at $60{ }^{\circ} \mathrm{C} .{ }^{b}$ Conversions were measured by GC. ${ }^{c}$ Identified by GC-MS; nd-not determined. ${ }^{d}$ Reaction performed at $40{ }^{\circ} \mathrm{C}$. 
These examples demonstrates the superior effect Pd nanoparticles immobilized onto the green support. The high reactivity aspect makes one believe that Pd nanoparticles on the cellulosic support effectively suppress the agglomeration of the bear surface nanoparticle, assisting in achieving rather high catalytic activity in comparison to the similar Pd-supported systems. ${ }^{54,55}$ The exclusive selectivity towards py-THQ depends on the interaction of the oraganonitrogen with the metal center in the strip. ${ }^{56}$ Availability of the large number of aliphatic $1^{\circ}$ and $2^{\circ}$ hydroxyl group on the cellulosic jute stick also affect the instantaneous microenvironment around the metal centers benignly. ${ }^{57}$ The possibility of hydrogen bonding interaction mediating the heterocyclic ring hydrogenation faster than that of the carbocyclic ring of the substrate, resulting exclusive selectivity towards py-THQ, cannot be ruled out.

\section{Recyclability studies}

One of the extraordinary feature of "dip-catalyst" is the ease of deployment in the system and its effortless post-reaction removal. Hence, reusability of spent catalyst in subsequent cycles for transfer hydrogenation of quinoline under optimized reaction condition is possible. To investigate its robustness and sustainability, the catalyst were extracted from the reaction mixture, thoroughly washed with water and dichloromethane and, dried for use in next cycle with fresh batch of quinoline as the substrate and THDB as reductant in aqueous medium. This procedure was repeated multiple times and catalyst was used in 32 consecutive cycles with excellent catalytic propensity without appreciable degradation of catalytic activity (Fig. 8a). This was corroborated by ICP-OES examination; samples were extracted at certain intervals and analyzed to determine if any Pd was leached out. As can be seen from Fig. 8b, no trace of Pd was detected in the solution. The surface morphology and stability of the recycled catalyst showed no severe damage to the jutestick surface or no agglomeration of the Pd nanoparticles (see ESI $\dagger$ ). The XPS analysis revealed that the peak at $335.4 \mathrm{eV}$ and $340.6 \mathrm{eV}$ reflect the preservation of $\mathrm{Pd}^{0}$ oxidation state. A small shoulder at $336.4 \mathrm{eV}$ corresponding to PdO could be ascribed to be due to long exposure to aerobic condition.

From the foregoing discussion, it appears that the excellent survivability of the catalyst comes from strong and appropriate bonding with huge number of hydroxyls groups on the support.
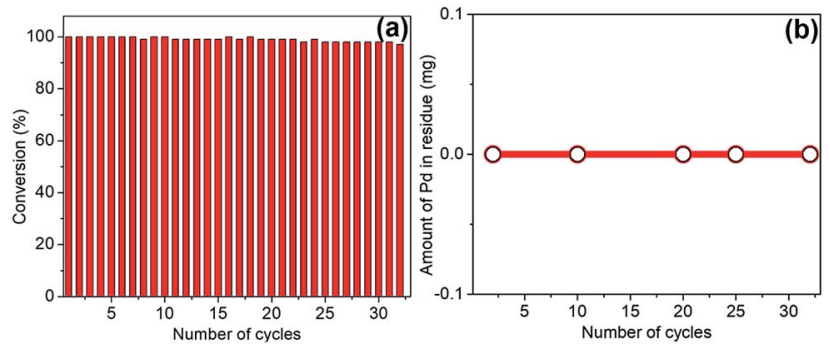

Fig. 8 (a) Reusability potential of the catalyst for quinoline hydrogenation in water using THDB reductant. (b) ICP-OES data showing the evidence of no leaching of $\mathrm{Pd}$ from the dip-catalyst after a large number of cycles.

\section{Conclusions}

Dip catalyst based on Pd nanoparticles supported on the naturally-occurring surface architecture of jute-stick was fabricated for its excellent transfer hydrogenation activity under mild conditions in water. The natural cellulosic surface provided favorable interaction for Pd nanoparticles. This dip catalyst was gainfully employed for chemoselective transfer hydrogenation of olefin with excellent conversion and exceptional organic functional group tolerance using THDB, and provided very high TOF. Quinoline and other $N$-heteroarenes were selectively and quantitatively hydrogenated at $60^{\circ} \mathrm{C}$ within a very short span of time ( $c f .40 \mathrm{~min}$ ) under atmospheric condition. The catalyst was found to possess impressive stability and could be used over 32 consecutive cycles without adversely affecting its original reactivity or surface morphology and/or chemical composition. The unique advantage of effortless catalyst monitoring, insertion or removal between the cycles were demonstrated. The use of this naturally-occurring support, ease of handling, low cost fabrication, convenient deployment, favorable organic functional groups on support to provide enhanced stability, impressive conversion and remarkable chemoselectivity, use of water as reaction medium and exceptional reusability may lead to commercialization of this catalyst for industrial applications. The environmentally benign catalyst fabrication strategy and remarkable catalytic transfer hydrogenations activity for a series of olefins and $\mathrm{N}$ heteroarenes offers a new route to develop catalysts with other metal nanoparticles for various catalytic transformations. Currently some such catalytic reactions are under study in our laboratory.

\section{Conflicts of interest}

There are no conflicts to declare.

\section{Acknowledgements}

MNS gratefully acknowledges the National Plan for Science, Technology and Innovation (MAARIFAH)-King Abdulaziz City for Science and Technology through the Science and Technology (KACST) Unit at King Fahd University of Petroleum and Minerals (KFUPM), the Kingdom of Saudi Arabia, award number 15-NAN4650-04. The author also gratefully acknowledges Dr Md. Abdul Aziz for providing the jute sticks.

\section{Notes and references}

1 Z. Zhang, N. A. Butt and W. Zhang, Chem. Rev., 2016, 116, 14769-14827.

2 P. Etayoa and A. Vidal-Ferran, Chem. Soc. Rev., 2013, 42, 728754.

3 N. M. Rezayee, D. C. Samblanet and M. S. Sanford, ACS Catal., 2016, 6, 6377-6383.

4 J. Yuwen, S. Chakraborty, W. W. Brennessel and W. D. Jones, ACS Catal., 2017, 7, 3735-3740. 
5 M. Shevlin, M. R. Friedfeld, H. Sheng, N. A. Pierson, J. M. Hoyt, L.-C. Campeau and P. J. Chirik, J. Am. Chem. Soc., 2016, 138, 3562-3569.

6 Y. Duan, L. Li, M.-W. Chen, C.-B. Yu, H.-J. Fan and Y.-G. Zhou, J. Am. Chem. Soc., 2014, 136, 7688-7700.

7 Y. T. Xia, J. Ma, X. D. Wang, L. Yanga and L. Wu, Catal. Sci. Technol., 2017, 7, 5515-5520.

8 X. Zhang, L. J. Durndell, M. A. Isaacs, C. M. A. Parlett, A. F. Lee and K. Wilson, ACS Catal., 2016, 6, 7409-7417.

9 J. Wen, R. Tan, S. Liu, Q. Zhao and X. Zhang, Chem. Sci., 2016, 7, 3047-3051.

10 A. Vandekerkhove, L. Claes, F. De Schouwer, C. V. Goethem, I. F. J. Vankelecom, B. Lagrain and D. E. De Vos, ACS Sustainable Chem. Eng., 2018, 6, 9218-9228.

11 X. C. Zhang, Y. H. Hu, C. F. Chen, Q. Fang, L. Y. Yang, Y. B. Lu, L. J. Xie, J. Wu, S. Li and W. Fang, Chem. Sci., 2016, 7, 4594-4599.

12 H. Konnerth and M. H. G. Prechtl, Green Chem., 2017, 19, 2762-2767.

13 S. Chakraborty and H. Berke, ACS Catal., 2014, 4, 2191-2194. 14 D. Wang and D. Astruc, Chem. Rev., 2015, 115, 6621-6686.

15 R. A. W. Johnstone, A. H. Wilby and I. D. Entwistle, Chem. Rev., 1985, 85, 129-170.

16 A. Vasseur, R. Membrat, D. Palpacelli, M. Giorgi, D. Nuel, L. Giordano and A. Martinez, Chem. Commun., 2018, 54, 10132-10134.

17 P. Sharma and Y. Sasson, Green Chem., 2019, 21, 261-267.

18 Y. Song, H. Wang, S. Liang, Y. Yu, L. Li and L. Wu, J. Catal., 2018, 361, 105-115.

19 N. Mrsaic, L. Lefort, J. A. F. Boogers, A. J. Minnaard, B. L. Feringa and J. G. de Vries, Adv. Synth. Catal., 2008, 350, 1081-1089.

20 M. Cerro-Alarcón, A. Maroto-Valiente, I. Rodríguez-Ramos and A. Guerrero-Ruiz, Carbon, 2005, 43, 2711-2722.

21 A. R. McDonald, C. Müller, D. Vogt, G. P. M. van Klinka and G. van Koten, Green Chem., 2008, 10, 424-432.

22 Z. An, J. He, Y. Dai, C. Yu, B. Li and J. He, J. Catal., 2014, 317, 105-113.

23 X. Chena, W. Wang, H. Zhu, W. Yanga and Y. Ding, J. Mol. Catal., 2018, 456, 49-56.

24 P. Ju, S. Wu, Q. Su, X. Li, Z. Liu, G. Lib and Q. Wu, J. Mater. Chem. A, 2019, 7, 2660-2664.

25 A. W. Petrov, D. Ferri, O. Kröcher and J. A. van Bokhoven, ACS Catal., 2019, 9, 2303-2312.

26 J. Lee, Y. S. Ryou, S. Hwang, Y. Kim, S. J. Cho, H. Lee, C. H. Kim and D. H. Kim, Catal. Sci. Technol., 2019, 9, 163-168.

27 T. Guo, X. Niec, J. Dub and J. Li, Chem. Eng. J., 2019, 361, 1345-1351.

28 K. Yakabi, A. Jones, A. Buchard, A. Roldan and C. Hammond, ACS Sustainable Chem. Eng., 2018, 6, 16341-16351.

29 M. N. Shaikh, M. A. Aziz, A. N. Kalanthoden, A. Helal, A. S. Hakeem and M. Bououdina, Catal. Sci. Technol., 2018, 8, 4709-4717.

30 R. Abu-Reziq, H. Alper, D. Wang and M. L. Post, J. Am. Chem. Soc., 2006, 128, 5279-5282.

31 G. Sun, J. An, H. Hu, C. Li, S. Zuo and H. Xia, Catal. Sci. Technol., 2019, 9, 1238-1244.
32 W. Dong, L. Zhang, C. Wang, C. Feng, N. Shang, S. Gao and C. Wang, RSC Adv., 2016, 6, 37118-37123.

33 J. Li, X. Zhou, N.-Z. Shang, C. Feng, S.-T. Gao and C. Wang, New J. Chem., 2018, 42, 16823-16828.

34 X. Xu, Y. Li, Y. Gong, P. Zhang, H. Li and Y. Wang, J. Am. Chem. Soc., 2012, 134, 16987-16990.

35 Y. Gong, P. Zhang, X. Xu, Y. Li, H. Li and Y. Wang, J. Catal., 2013, 297, 272-280.

36 Z. Xiang, Y. Chen, Q. Liu and F. Lu, Green Chem., 2018, 20, 1085-1094.

37 V. Sharma, A. Bahuguna and V. Krishnan, Adv. Mater. Interfaces, 2017, 4, 1700604.

38 E. Hariprasad and T. P. Radhakrishnan, Chem.-Eur. J., 2010, 16, 14378-14384.

39 E. Hariprasad and T. P. Radhakrishnan, ACS Catal., 2012, 2, 1179-1186.

40 G. C. Zheng, L. Polavarapu, L. M. Liz-Marzan, I. Pastoriza- Santos and J. Perez-Juste, Chem. Commun., 2015, 51, 4572-4575.

41 V. W. Faria, D. G. Oliveira, M. H. Kurz, F. F. Gonçalves, C. W. Scheeren and G. R. Rosa, RSC Adv., 2014, 4, 13446-13451.

42 M. N. Shaikh, A. Helal, A. N. Kalanthoden, B. Najjar, M. A. Aziz and H. D. Mohamed, Catal. Commun., 2019, 119, 134-138.

43 M. N. Shaikh, M. A. Aziz, A. Helal, M. Bououdina, Z. H. Yamani and T.-J. Kim, RSC Adv., 2016, 6, 41687-41695. 44 M. N. Shaikh, M. Bououdina, A. A. Jimoh, M. A. Aziz, A. Helal, A. S. Hakeem, Z. H. Yamani and T.-J. Kim, New J. Chem., 2015, 39, 7293-7299.

45 L. Nayak, A. Lakshmanan and D. P. Ray, J. Indian Chem. Soc., 2012, 89, 1723-1727.

46 T. Teranishi and M. Miyak, Chem. Mater., 1998, 10, 594-600. 47 F. G. de Rivera, I. Angurell, M. D. Rossell, R. Erni, J. Llorca, N. J. Divins, G. Muller, M. Seco and O. Rossell, Chem.-Eur. J., 2013, 19, 11963-11974.

48 S. Y. Xu, X. P. Yan, Q. Bu and H. A. Xia, Cellulose, 2017, 24, 2403-2413.

49 G. Zheng, K. Kaefer, S. Mourdikoudis, L. Polavarapu, B. Vaz, S. E. Cartmell, A. Bouleghlimat, N. J. Buurma, L. Yate, A. R. de Lera, L. M. Liz-Marzán, I. Pastoriza-Santos and J. Pérez-Juste, J. Phys. Chem. Lett., 2015, 6, 230-238.

50 H. Tian and J. He, Langmuir, 2016, 32, 12269-12282.

51 A. Goswami, A. K. Rathi, C. Aparicio, O. Tomanec and M. Petr, ACS Appl. Mater. Interfaces, 2017, 9, 2815-2824.

52 R. L. Halterman, in Comprehensive Asymmtric Catalysis, ed. E. N. Jacobson, A. Paltz and H. Yamamoto, Springer, New York, 1999, p. 183.

53 S. P. Cummings, T.-N. Le, G. E. Fernandez, L. G. Quiambao and B. J. Stokes, J. Am. Chem. Soc., 2016, 138, 6107-6110.

54 Q. Xuan and Q. Song, Org. Lett., 2016, 18, 4250-4253.

55 J. Wang, Y.-L. Qin, X. Liu and X.-B. Zhang, J. Mater. Chem., 2012, 22, 12468-12470.

56 R. H. Fish, E. Baralt and S. J. Smith, Organometallics, 1991, 10, 54-56.

57 Y. Zhang, M. E. Grass, S. E. Habas, F. Tao, T. Zhang, P. Yang and G. A. Somorjai, J. Phys. Chem. C, 2007, 111, 12243-12253. 